\title{
Études/Inuit/Studies
}

\section{NAPPAALUK, Mitiarjuk, 2002 Sanaaq, roman translittéré et traduit de l'inuktitut au français par Bernard Saladin d'Anglure, préface et postface de Bernard Saladin d'Anglure, Québec, Stanké, 303 pages.}

\section{Cécile Pachocinski}

Volume 28, numéro 2, 2004

\section{Espaces-Lieux-Noms}

Spaces-Places-Names

URI : https://id.erudit.org/iderudit/013215ar

DOI : https://doi.org/10.7202/013215ar

Aller au sommaire du numéro

\section{Éditeur(s)}

Association Inuksiutiit Katimajiit Inc.

Centre interuniversitaire d'études et de recherches autochtones (CIÉRA)

\section{ISSN}

0701-1008 (imprimé)

1708-5268 (numérique)

Découvrir la revue

Citer ce compte rendu

Pachocinski, C. (2004). Compte rendu de [NAPPAALUK, Mitiarjuk, 2002 Sanaaq, roman translittéré et traduit de l'inuktitut au français par Bernard Saladin d'Anglure, préface et postface de Bernard Saladin d'Anglure, Québec, Stanké, 303 pages.] Études/Inuit/Studies, 28(2), 245-247. https://doi.org/10.7202/013215ar d'utilisation que vous pouvez consulter en ligne. 
SIMARD, Jean-Jacques

1983 Par delà le Blanc et le mal. Rapports identitaires et colonialisme au pays des Inuit, Sociologie et sociétés, 15(2): 55-71.

Marcelle Chabot 49, chemin du Village Pontiac (Québec) J0X 2G0

Canada marcelle_chabot@hotmail.com

\begin{abstract}
NAPPAALUK, Mitiarjuk
2002 Sanaaq, roman translittéré et traduit de l'inuktitut au français par Bernard Saladin d'Anglure, préface et postface de Bernard Saladin d'Anglure, Québec, Stanké, 303 pages.
\end{abstract}

La préface du roman reprend succinctement le contexte qui lui a donné le jour. Bernard Saladin d'Anglure y présente l'auteure, son amie et informatrice Mitiarjuk Nappaaluk qui est aujourd'hui reconnue comme une figure importante de Kangirsujuaq (Nunavik). Ce roman est le résultat d'une double sollicitation. Dans le début des années 1950, le Père Robert Lechat demande à l'auteure de transcrire le «plus grand nombre possible de termes et de formes grammaticales» (p. 8) pour apprendre l'inuktitut. L'autre demande est celle de l'anthropologue Bernard Saladin d'Anglure. Lorsqu'il découvre la première partie du manuscrit en 1956, il encourage Mitiarjuk Nappaaluk à en écrire la suite entre 1967 et 1969, soit les épisodes 38 à 48. Cette édition en français permet de faire connaître à un large public un récit qui jusque-là n'avait été accessible qu'aux Inuit et à une communauté restreinte de chercheurs. La première publication du texte remonte à l'année 1970 avec la thèse de doctorat de Bernard Saladin d'Anglure qui porte directement sur ce roman et les commentaires apportés par son auteure. Par la suite, en 1984, il rend possible la publication du roman en écriture syllabique normalisée et c'est en 2002 que paraît le roman en français dans une édition grand public.

Le roman est composé de 48 épisodes. Il raconte la vie quotidienne d'une famille inuit, celle de Sanaaq, au moment de la période précédant et commençant la transition de la vie semi-nomade à la vie dans les maisons fabriquées. Celle-ci correspond à l'enfance de Mitiarjuk Nappaaluk, entre sa naissance en 1930 et les temps suivant la seconde guerre mondiale. Les épisodes très courts et les titres qui les accompagnent ne structurent pas à eux seuls l'histoire racontée. Inutile de chercher une unité d'action et de temps par épisode, comme nous y sommes parfois habitués avec la structure par chapitres. Ce sont tout autant les différents rythmes saisonniers par le flux et le reflux des saisons et du gibier, les rythmes journaliers par la périodicité du jour et de la nuit, 
ou encore les indications qui portent sur les personnages qui vieillissent, comme les enfants de Sanaaq qui permettent au lecteur de se repérer dans le temps.

Bien que les personnages soient nombreux: une trentaine, on se resitue assez facilement dans l'histoire qui suit de très près les activités de Sanaaq et de ses proches: sa fille Qumaq, son mari Qalingu et leur fils Irsutuq, sa sœur Arnatuinnaq qui sont, en gros, les principaux personnages.

Les thèmes importants du roman tournent autour de la vie quotidienne de cette famille. On peut noter, entre autres, les activités de subsistance (chasse, pêche, cueillette, ramassage des œufs), de préparation et de consommation de la nourriture (cuisine, repas, préparation des caches), mais aussi les contacts de cette famille avec les missionnaires et l'administration des «Blancs». On retrouve ces thèmes au fil du récit, certains se répétant plus que d'autres. Les répétitions et l'étonnante sobriété de l'action narrée donnent une marque distinctive au roman. Loin d'être lassantes pour le lecteur, elles sont d'une grande richesse pour la compréhension de la vie quotidienne des personnages et de leurs relations sociales.

Les émotions et les différentes sensations éprouvées par les personnages participent pleinement au portrait de l'univers quotidien, comme l'a souligné Bernard Saladin d'Anglure dans la postface (p. 279). Le caractère très vivant du roman est rendu par de nombreuses références aux sensations. Les quelques exemples que nous avons notés se rapportent au froid, au chaud associé à une sensation désagréable lorsque que Sanaaq séjourne à hôpital dans le Sud et aux odeurs de nourriture, comme celle du phoque ou encore à la mauvaise odeur alliée à la possession de Maatiusi par une femme invisible. On trouve également de nombreuses références à l'ouïe par les onomatopées, ou encore à la vue qui est extrêmement présente dans les scènes de chasse, de cueillette et de pêche. Ces indications sur les émotions et les sens permettent au lecteur d'entrer dans la compréhension de l'expérience vécue, de ce qui est agréable et de ce qui ne l'est pas, de ce qui est valorisé et de ce qui ne l'est pas, de ce qui est toléré et de ce qui ne l'est pas. Plus que des effets de réel, elles engagent le lecteur dans une vision intime de la culture inuit reconstruite ici par Mitiarjuk Nappaaluk par l'écriture de fiction.

Dans la postface, Bernard Saladin d'Anglure commente brièvement «la forme et le style du roman», «le fond de l'œuvre et le non-dit qui l'entoure», «le contexte historique et culturel du roman» et en dernier lieu, «les personnages du récit». Ces pages précieuses donnent au lecteur des pistes de lecture que l'on aimerait encore plus abondantes. Non pas que la lecture «nue» du roman ne se suffise pas en elle-même, mais surtout parce qu'elles donnent au lecteur le goût d'en savoir plus. Cependant, on ne tiendra pas rigueur de la présence discrète de l'initiateur de cette publication. Par ce choix, il est manifeste qu'il a souhaité donner toute la place à la parole des personnages et à l'écriture de l'auteure. La postface et le glossaire permettent ainsi de se repérer autant dans les termes en inuktitut que dans les noms des personnages, et ajoutent au plaisir de la lecture. 
Ce récit est sans conteste une référence majeure de la littérature inuit actuelle tant pour les Inuit que pour le public extérieur. Cette publication va de pair avec la reconnaissance qui est accordée aujourd'hui à son auteure Mitiarjuk Nappaaluk au Québec, au Canada et sur le plan international. Ce roman nous apparaît comme un incontournable pour les chercheurs travaillant avec les Inuit et un merveilleux livre d'introduction à la culture inuit pour les profanes.

Cécile Pachocinski

CIERA

Université Laval

Québec (Québec) G1K 7P4

Canada

cecile.pachocinski@ciera.ulaval.ca 